J. Appl. Numer. Optim. 3 (2021), No. 3, pp. 501-512

Available online at http://jano.biemdas.com

https://doi.org/10.23952/jano.3.2021.3.05

\title{
ON SEQUENTIAL OPTIMALITY THEOREMS FOR LINEAR FRACTIONAL OPTIMIZATION PROBLEMS INVOLVING INTEGRAL FUNCTIONS DEFINED $\mathbf{O N} L_{n}^{2}[0,1]$
}

\author{
MOON HEE KIM ${ }^{1}$, GWI SOO KIM ${ }^{2}$, GUE MYUNG LEE ${ }^{2, *}$ \\ ${ }^{1}$ College of General Education, Tongmyong University, Busan 48520, Korea \\ ${ }^{2}$ Department of Applied Mathematics, Pukyong National University, Busan 48513, Korea
}

\begin{abstract}
We consider a linear fractional optimization problem involving integral functions defied on $L_{n}^{2}[0,1]$, and obtain sequential optimality conditions for the linear fractional optimization problem which hold without any constraint qualification, and are expressed by sequences. By using the optimality theorems, we formulate the nonfractional dual problem for the linear fractional optimization problem, and prove the weak duality theorem and the strong duality theorem.
\end{abstract}

Keywords. Duality theorem; Linear fractional optimization problem; Sequential optimality theorem; Constraint qualification.

\section{INTRODUCTION AND PRELIMINARIES}

Jeyakumar et al. [1] obtained the sequential Lagrange multiplier optimality conditions for convex optimization problems, which held without any constraint qualification, and were expressed by sequences. Such sequential optimality conditions have been investigated for many kinds of convex optimization problems recently; see, e.g., [2, 3, 4, 5]. Using variable transformation, Craven [6] and Craven et al. [7] obtained the equivalent nonfractional linear optimization problem from a linear fractional optimization problem. They formulated the dual problem by using the equivalent problem, and then gave duality theorems for the primal problem and its dual problem. So the dual problem is a nonfractional dual one for the linear fractional optimization problem which the duality theorems hold. By using the approach of Craven [6] and Craven et al. [7], Kim et al. formulated the dual problem for a semidefinite linear fractional optimization problem, and then proved directly duality theorems, which hold under constraint qualification [8], and without any constraint qualification [9]. Kim et al. [10] formulated the dual problem for a second-order cone linear fractional optimization problem, which was expressed by sequences, and then proved the weak duality theorem and the strong duality theorem which hold between the primal problem and its dual problem. The strong duality theorem held without any constraint qualification.

\footnotetext{
${ }^{*}$ Corresponding author.

E-mail addresses: mooni@tu.ac.kr (M.H. Kim), gwisoo1103@hanmail.net (G.S. Kim), gmlee@pknu.ac.kr (G.M. Lee).

Received October 15, 2021; Accepted November 23, 2021.
}

(C)2021 Journal of Applied and Numerical Optimization 
Consider the following linear fractional optimization problem:

$$
\begin{aligned}
\text { Minimize } & \frac{\int_{0}^{1} c(t)^{T} x(t) d t+\alpha}{\int_{0}^{1} d(t)^{T} x(t) d t+\beta} \\
\text { subject to } \quad & x(\cdot) \in K, \\
& \int_{0}^{1} a_{i}(t)^{T} x(t) d t=b_{i}, i=1, \cdots, m,
\end{aligned}
$$

where $c, d \in L_{n}^{2}[0,1], a_{i} \in L_{n}^{2}[0,1], b_{i} \in \mathbb{R}, i=1, \cdots, m, \alpha$ and $\beta$ are two given real number, and $K$ is a closed convex cone in $L_{n}^{2}[0,1]$.

Let $\triangle=\left\{x \in L_{n}^{2}[0,1] \mid x \in K, \int_{0}^{1} a_{i}(t)^{T} x(t) d t=b_{i}, i=1, \cdots, m\right\}$. We define the nonnegative dual cone of $K$ as $K^{*}=\left\{z \in L_{n}^{2}[0,1] \mid \int_{0}^{1} z(t)^{T} x(t) d t \geqq 0 \quad \forall x \in L_{n}^{2}[0,1]\right\}$. We recall that $x=y$ in $L_{n}^{2}[0,1]$ if and only if $x(t)=y(t)$ a.e on $[0,1]$. We define the inner product $\langle\cdot, \cdot\rangle$ as $\langle f, g\rangle=$ $\int_{0}^{1} f(t) g(t) d t$ for any $f, g \in L_{n}^{2}[0,1]$. Then $L_{n}^{2}[0,1]$ is a Hilbert space with the inner product.

Recently, Kim et al. [8] formulated the nonfractional dual problem for $(\mathrm{P})$ under a constraint qualification, and proved the weak duality theorem, the strong duality theorem, and the converse duality theorem which held between $(\mathrm{P})$ and the dual problem under the constraint qualification.

In this paper, we obtain sequential optimality theorems for $(\mathrm{P})$ which hold without any constraint qualification and are expressed by sequences. By using the optimality theorems, we formulate the nonfractional dual problem (D) for $(\mathrm{P})$, which are presented with limits, and prove the weak duality theorem and the strong duality theorem which hold between (P) and (D).

\section{Sequential Optimality theorems}

We give the definition of the convex function and the conjugate function, and then state the well-known results about the epigraphs of the conjugate functions of the convex functions.

Let $E$ be a Banach space with norm $x \mapsto\|x\|$, and let $E^{*}$ the dual of $E$.

Definition 2.1. The conjugate function of a function $f: E \rightarrow \mathbb{R} \cup\{+\infty\}$ is the function $f^{*}$ : $E^{*} \rightarrow \mathbb{R} \cup\{+\infty\}$ defined by

$$
f^{*}\left(x^{*}\right):=\sup _{x \in E}\left\{\left\langle x^{*}, x\right\rangle-f(x)\right\}\left(x^{*} \in E^{*}\right) .
$$

A function $g: E \rightarrow \mathbb{R} \cup\{+\infty\}$ is said to be convex if, for all $t \in[0,1]$,

$$
g((1-t) x+t y) \leq(1-t) g(x)+\operatorname{tg}(y)
$$

for all $x, y \in E$. Let $g: E \rightarrow \mathbb{R} \cup\{+\infty\}$ be a proper convex function. We denote the epigraph of $g$ by epi $g$, that is, epi $g:=\{(x, r) \in E \times \mathbb{R}: g(x) \leq r\}$.

We say a function $g$ is lower semicontinuous if $\liminf _{y \rightarrow x} g(y) \geq g(x)$ for all $x \in E$.

The following two propositions are well known (see [11] and [12]).

Proposition 2.1. Let $E$ be a Banach space. Consider a family of proper lower semicontinuous convex functions $\phi_{i}: E \rightarrow \mathbb{R} \cup\{+\infty\}, i \in I$, where $I$ is an arbitrary index set. Suppose that $\sup _{i \in I} \phi_{i}$ is not identically $+\infty$. Then

$$
\operatorname{epi}\left(\sup _{i \in I} \phi_{i}\right)^{*}=\operatorname{clco} \bigcup_{i \in I} \operatorname{epi} \phi_{i}^{*} .
$$


Proposition 2.2. Let $E$ be a Banach space. Let $\phi_{1}: E \rightarrow \mathbb{R}, \phi_{2}: E \rightarrow \mathbb{R} \cup\{+\infty\}$ be proper, lower semicontinuous convex functions. Then

$$
\operatorname{epi}\left(\phi_{1}+\phi_{2}\right)^{*}=\operatorname{cl}\left(\text { epi } \phi_{1}^{*}+\text { epi } \phi_{2}^{*}\right) \text {. }
$$

In addition, if one of the proper, lower semicontinuous convex functions is continuous, then

$$
\operatorname{epi}\left(\phi_{1}+\phi_{2}\right)^{*}=\operatorname{epi} \phi_{1}^{*}+\text { epi } \phi_{2}^{*}
$$

Now we give a sequential optimality theorem for $(\mathrm{P})$ which hold without any constraint qualification.

Theorem 2.1. Let $\bar{x} \in \triangle$. Assume that for any $x \in \triangle, \int_{0}^{1} d(t)^{T} x(t)+\beta>0$. Then the following are equivalent:

(i) $\bar{x}$ is an optimal solution of $(P)$;

(ii) $(0,0) \in\{(c-q(\bar{x}) d,-\alpha+q(\bar{x}) \beta)\}+c l\left(\bigcup_{\lambda_{i} \in \mathbb{R}} \sum_{i=1}^{m} \lambda_{i}\left(a_{i}, b_{i}\right)+\left(-K^{*}\right) \times \mathbb{R}_{+}\right)$, where $q(\bar{x})=\frac{\int_{0}^{1} c(t)^{T} \bar{x}(t) d t+\alpha}{\int_{0}^{1} d(t)^{T} \bar{x}(t) d t+\beta}$

(iii) there exist $\lambda_{i}^{n} \in \mathbb{R}, i=1, \cdots, m$ and $k_{n}^{*} \in K^{*}$ such that

$$
\begin{aligned}
& c-q(\bar{x}) d+\lim _{n \rightarrow \infty}\left(\sum_{i=1}^{m} \lambda_{i}^{n} a_{i}-k_{n}^{*}\right)=0 \\
& \text { and } \lim _{n \rightarrow \infty} \int_{0}^{1} k_{n}^{*}(t)^{T} \bar{x}(t) d t=0 .
\end{aligned}
$$

Proof. Let

$$
f(x)=\int_{0}^{1} c(t)^{T} x(t) d t+\alpha-q(\bar{x})\left[\int_{0}^{1} d(t)^{T} x(t) d t+\beta\right]
$$

and

$$
h_{i}(x)=\int_{0}^{1} a_{i}(t)^{T} x(t) d t-b_{i}, i=1, \cdots, m .
$$

Then $f: L_{n}^{2}[0,1] \rightarrow \mathbb{R}$, and $h_{i}: L_{n}^{2}[0,1] \rightarrow \mathbb{R}, i=1, \cdots, m$ are continuous and affine. Let

$$
D=\left\{x \in L_{n}^{2}[0,1] \mid h_{i}(x)=0, i=1, \cdots, m\right\} .
$$

Then $\Delta=D \cap K$. Notice that $\bar{x}$ is an optimal solution of (P) if and only if $f(x)+\delta_{\triangle}(x) \geqq$ $f(\bar{x})+\delta_{\triangle}(\bar{x})=0$ for any $x \in L_{n}^{2}[0,1]$, that is, $\left(f+\delta_{\triangle}\right)^{*}(0) \leq 0$, where $\delta_{\triangle}$ is the indicator of $\triangle$. Let $\bar{x}$ be an optimal solution of (P). By Proposition 2.2, we have

$$
\begin{aligned}
(0,0) & \in \operatorname{epi}\left(f+\delta_{\triangle}\right)^{*} \\
& =\text { epi } f^{*}+\operatorname{epi} \delta_{\triangle}^{*} \\
& =\text { epi } f^{*}+\operatorname{cl}\left(\text { epi } \delta_{D}^{*}+\text { epi } \delta_{K}^{*}\right) .
\end{aligned}
$$

Thus

$$
(0,0) \in \operatorname{epi} f^{*}+\operatorname{cl}\left(\operatorname{epi} \delta_{D}^{*}+\operatorname{epi} \delta_{K}^{*}\right)
$$


Since

$$
\begin{aligned}
f^{*}\left(v^{*}\right) & =\sup \left\{\int_{0}^{1} v^{*}(t)^{T} x(t) d t-f(x) \mid x \in L_{n}^{2}[0,1]\right\} \\
& =\sup \left\{\int_{0}^{1}\left[v^{*}(t)-c(t)+q(\bar{x}) d(t)\right]^{T} x(t) d t \mid x \in L_{n}^{2}[0,1]\right\}-\alpha+q(\bar{x}) \beta \\
& = \begin{cases}-\alpha+q(\bar{x}) \beta, & \text { if } v^{*}=c-q(\bar{x}) d, \\
+\infty, & \text { if } v^{*} \neq c-q(\bar{x}) d,\end{cases}
\end{aligned}
$$

and epi $f^{*}=\{(c-q(\bar{x}) d,-\alpha+q(\bar{x}) \beta)\}+\{0\} \times \mathbb{R}_{+}$. Moreover, epi $\delta_{K}^{*}=\left(-K^{*}\right) \times \mathbb{R}_{+}$. Since $\delta_{D}(x)=\sup _{\lambda_{i} \in \mathbb{R}}\left(\sum_{i=1}^{m} \lambda_{i} h_{i}\right)(x)$, it follows from Proposition 2.2 that

$$
\begin{aligned}
\operatorname{epi} \delta_{D}^{*} & =\operatorname{clco} \bigcup_{\lambda_{i} \in \mathbb{R}} \operatorname{epi}\left(\sum_{i=1}^{m} \lambda_{i} h_{i}\right)^{*} \\
& =\operatorname{cl} \bigcup_{\lambda_{i} \in \mathbb{R}} \operatorname{epi}\left(\sum_{i=1}^{m} \lambda_{i} h_{i}\right)^{*} \\
& =\operatorname{cl} \bigcup_{\lambda_{i} \in \mathbb{R}} \sum_{i=1}^{m} \operatorname{epi}\left(\lambda_{i} h_{i}\right)^{*}
\end{aligned}
$$

and

$$
\begin{aligned}
\left(\lambda_{i} h_{i}\right)^{*}\left(v^{*}\right) & =\sup \left\{\left\langle v^{*}, x\right\rangle-\left(\lambda_{i} h_{i}\right)(x) \mid x \in L_{n}^{2}[0,1]\right\} \\
& =\sup \left\{\left\langle v^{*}, x\right\rangle-\lambda_{i} \int_{0}^{1} a_{i}(t)^{T} x(t) d t \mid x \in L_{n}^{2}[0,1]\right\} \\
& =\sup \left\{\int_{0}^{1}\left[v^{*}(t)-\lambda_{i} a_{i}(t)\right]^{T} x(t) d t \mid x \in L_{n}^{2}[0,1]\right\} \\
& =\left\{\begin{array}{l}
\lambda_{i} b_{i}, \text { if } v^{*}=\lambda_{i} a_{i}, \\
+\infty, \text { if } v^{*} \neq \lambda_{i} a_{i} .
\end{array}\right.
\end{aligned}
$$

So,

$$
\begin{aligned}
\operatorname{epi}\left(\lambda_{i} h_{i}\right)^{*} & =\left\{\left(\lambda_{i} a_{i}, \lambda_{i} b_{i}\right)\right\}+\{0\} \times \mathbb{R}_{+} \\
& =\left\{\lambda_{i}\left(a_{i}, b_{i}\right)\right\}+\{0\} \times \mathbb{R}_{+} .
\end{aligned}
$$

Hence, epi $\delta_{D}^{*}=\operatorname{cl}\left(\bigcup_{\lambda_{i} \in \mathbb{R}} \sum_{i=1}^{m}\left\{\lambda_{i}\left(a_{i}, b_{i}\right)\right\}+\{0\} \times \mathbb{R}_{+}\right)$. From (2.1), one has

$$
(0,0) \in\{(c-q(\bar{x}) d,-\alpha+q(\bar{x}) \beta)\}+\{0\} \times \mathbb{R}_{+}+\operatorname{cl}\left(\bigcup_{\lambda_{i} \in \mathbb{R}_{i=1}}^{m}\left\{\lambda_{i}\left(a_{i}, b_{i}\right)\right\}+\left\{-K^{*}\right\} \times \mathbb{R}_{+}\right) .
$$

Thus

$$
(0,0) \in\{(c-q(\bar{x}) d,-\alpha+q(\bar{x}) \beta)\}+\operatorname{cl}\left(\bigcup_{\lambda_{i} \in \mathbb{R}} \sum_{i=1}^{m}\left\{\lambda_{i}\left(a_{i}, b_{i}\right)\right\} \quad+\left(-K^{*}\right) \times \mathbb{R}_{+}\right) .
$$

Hence (ii) holds. From (2.2), there exist $\lambda_{i}^{n} \in \mathbb{R}$ and $k_{n} \in K^{*}, r_{n} \in \mathbb{R}_{+}$such that

$$
0=c-q(\bar{x}) d+\lim _{n \rightarrow \infty}\left[\sum_{i=1}^{m} \lambda_{i}^{n} a_{i}-k_{n}^{*}\right]
$$


and

$$
0=-\alpha+q(\bar{x}) \beta+\lim _{n \rightarrow \infty}\left[\sum_{i=1}^{m} \lambda_{i}^{n} b_{i}+r_{n}\right]
$$

Hence, we have

$$
\begin{aligned}
& 0= \int_{0}^{1} c(t)^{T} \bar{x}(t) d t-q(\bar{x}) \int_{0}^{1} d(t)^{T} \bar{x}(t) d t \\
&+\lim _{n \rightarrow \infty} \int_{0}^{1}\left[\sum_{i=1}^{m} \lambda_{i}^{n} a_{i}^{T}-k_{n}^{*}(t)\right]^{T} \bar{x}(t) d t \\
&+\alpha-q(\bar{x}) \beta-\lim _{n \rightarrow \infty} \int_{0}^{1}\left[\sum_{i=1}^{m} \lambda_{i}^{n} b_{i}+r_{n}\right] \\
&=\quad \int_{0}^{1} c(t)^{T} \bar{x}(t) d t+\alpha-q(\bar{x})\left[\int_{0}^{1} d(t)^{T} \bar{x}(t) d t+\beta\right] \\
& \quad+\lim _{n \rightarrow \infty}\left[\sum_{i=1}^{m} \lambda_{i}^{n} \int_{0}^{1}\left(a_{i}^{T} \bar{x}(t)-b_{i}\right) d t-\int_{0}^{1} k_{n}^{*}(t)^{T} \bar{x}(t) d t-r_{n}\right] \\
&=\lim _{n \rightarrow \infty}\left[-\int_{0}^{1} k_{n}^{*}(t) \bar{x}(t) d t-r_{n}\right] .
\end{aligned}
$$

Since $\int_{0}^{1} k_{n}^{*}(t)^{T} \bar{x}(t) d t \geqq 0$ and $r_{n} \geqq 0, \lim _{n \rightarrow \infty} r_{n}=0$ and $\lim _{n \rightarrow \infty} \int_{0}^{1} k_{n}^{*}(t) \bar{x}(t) d t=0$. Thus there exist $\lambda_{i}^{n} \in \mathbb{R}, i=1, \cdots, m$ and $k_{n}^{*} \in K^{*}$ such that

$$
\begin{aligned}
& 0=c-q(\bar{x}) d+\lim _{n \rightarrow \infty}\left[\sum_{i=1}^{m} \lambda_{i}^{n} a_{i}-k_{n}^{*}\right] \text { and } \\
& 0=\lim _{n \rightarrow \infty} \int_{0}^{1} k_{n}^{*}(t)^{T} \bar{x}(t) d t .
\end{aligned}
$$

So (iii) holds.

Now we prove that (iii) implies the optimality of $\bar{x}$. Suppose that (iii) holds. Then, for any $x \in \triangle$,

$$
\begin{aligned}
0= & \int_{0}^{1}[c(t)-q(\bar{x}) d(t)]^{T}(x(t)-\bar{x}(t)) d t \\
& \quad+\lim _{n \rightarrow \infty} \sum_{i=1}^{m} \lambda_{i}^{n} \int_{0}^{1}\left[a_{i}(t)^{T}(x(t)-\bar{x}(t))-k_{n}(t)^{T}(x(t)-\bar{x}(t))\right] d t \\
= & \int_{0}^{1} c(t)^{T} x(t) d t+\alpha-q(\bar{x})\left[\int_{0}^{1} d(t)^{T} x(t) d t+\beta\right] \\
& \quad-\int_{0}^{1}\left[c(t)^{T} \bar{x}(t) d t+\alpha\right]+q(\bar{x})\left[\int_{0}^{1} d(t)^{T} \bar{x}(t) d t+\beta\right] \\
& \quad+\lim _{n \rightarrow \infty}\left[-\int_{0}^{1} k_{n}(t)^{T}(x(t)-\bar{x}(t)) d t\right] \\
= & \int_{0}^{1} c(t)^{T} x(t) d t+\alpha-q(\bar{x})\left[\int_{0}^{1} d(t)^{T} x(t) d t+\beta\right] \\
& \quad-\lim _{n \rightarrow \infty} \int_{0}^{1} k_{n}(t)^{T} x(t) d t .
\end{aligned}
$$


Since $\lim _{n \rightarrow \infty} \int_{0}^{1} k_{n}(t)^{T} x(t) d t \geqq 0$ for any $x \in \triangle$, we have

$$
\int_{0}^{1} c(t)^{T} x(t) d t+\alpha-q(\bar{x})\left[\int_{0}^{1} d(t)^{T} x(t) d t+\beta\right] \geqq 0 .
$$

In view of $\int_{0}^{1} d(t)^{T} x(t) d t+\beta>0$ for any $x \in \triangle$, we obtain

$$
q(\bar{x}) \leqq \frac{\int_{0}^{1} c(t)^{T} x(t) d t+\alpha}{\int_{0}^{1} d(t)^{T} x(t) d t+\beta} \quad \text { for any } x \in \triangle .
$$

Since $q(\bar{x})=\frac{\int_{0}^{1} c(t)^{T} \bar{x}(t) d t+\alpha}{\int_{0}^{1} d(t)^{T} \bar{x}(t) d t+\beta}$, we have that $\bar{x}$ is an optimal solution of (P).

From the proof of Theorem 2.1, we can obtain the following theorem.

Theorem 2.2. Let $\bar{x} \in \triangle$ and assume that, for any $x \in \triangle, \int_{0}^{1} d(t)^{T} x(t) d t+\beta>0$. Suppose that the set $\bigcup_{\lambda_{i} \in \mathbb{R}}\left\{\sum_{i=1}^{m} \lambda_{i}\left(a_{i}, b_{i}\right)\right\}+\left(-K^{*}\right) \times \mathbb{R}_{+}$is closed in $L_{n}^{2}[0,1] \times \mathbb{R}$. Then the following are equivalent:

(i) $\bar{x}$ is an optimal solution of $(P)$;

(ii) $(0,0) \in\{(c-q(\bar{x}) d,-\alpha+q(\bar{x}) \beta)\}+\bigcup_{\lambda_{i} \in \mathbb{R}}\left\{\sum_{i=1}^{m} \lambda_{i}\left(a_{i}, b_{i}\right)\right\}+\left(-K^{*}\right) \times \mathbb{R}_{+}$, where $q(\bar{x})=\frac{\int_{0}^{1} c(t)^{T} \bar{x}(t) d t+\alpha}{\int_{0}^{1} d(t)^{T} \bar{x}(t) d t+\beta}$

(iii) there exist $\lambda_{i} \in \mathbb{R}, i=1, \cdots, m$ and $k^{*} \in K^{*}$ such that

$$
\begin{aligned}
& c-q(\bar{x}) d+\left(\sum_{i=1}^{m} \lambda_{i} a_{i}-k^{*}\right)=0 \\
& \text { and } \int_{0}^{1} k^{*}(t)^{T} \bar{x}(t) d t=0 .
\end{aligned}
$$

Now we give an example that the set $\bigcup_{\lambda_{i} \in \mathbb{R}}\left\{\sum_{i=1}^{m} \lambda_{i}\left(a_{i}, b_{i}\right)\right\}+\left(-K^{*}\right) \times \mathbb{R}^{+}$may not be closed. This example is obtained by modifying a known example (https://math.stackexchange.com/ questions/712188/how-can-the-sum-of-two-closed-cones-be-not-closed).

Example 2.1. Let $K=\left\{\left(x_{1}, x_{2}, x_{3}\right) \in L_{3}^{2}[0,1] \mid x_{1}(t) \geqq \sqrt{x_{2}(t)^{2}+x_{3}(t)^{2}}\right.$ a.e. in $\left.[0,1]\right\}$. Then $K$ is a closed convex cone in $L_{3}^{2}[0,1]$. Let $a_{1}(t)=(1,0,-1)(t \in[0,1])$ and $b_{1}=0$. Consider the set $\bigcup_{\lambda \in \mathbb{R}} \lambda\left(a_{1}, b_{1}\right)+\left(-K^{*}\right) \times \mathbb{R}^{+}$. Suppose that $(0,-1,0,0) \in \bigcup_{\lambda \in \mathbb{R}}\left\{\lambda\left(a_{1}, b_{1}\right)\right\}+\left(-K^{*}\right) \times \mathbb{R}^{+}$. Then there exists $\lambda \in \mathbb{R}$ such that $(\lambda, 1,-\lambda) \in K^{*}$. Since $\Lambda:=\left\{\left(x_{1}, x_{2}, x_{3}\right)\right.$ in $\left.\mathbb{R}^{3} \mid x_{1} \geqq \sqrt{x_{2}^{2}+x_{3}^{2}}\right\} \subset$ $K$ and the nonnegative dual cone of $\Lambda$ is $\Lambda$ in $\mathbb{R}^{3},(\lambda, 1,-\lambda) \in \Lambda$ and so $\lambda^{2} \geqq 1^{2}+(-\lambda)^{2}$, which ia a contradiction. Hence $(0,-1,0,0) \notin \bigcup_{\lambda \in \mathbb{R}} \lambda\left(a_{1}, b_{1}\right)+\left(-K^{*}\right) \times \mathbb{R}^{+}$. But

$$
\begin{aligned}
(0,-1,0,0) & =\lim _{\lambda \rightarrow \infty}\left[\lambda(1,0,-1,0)+\left(-\sqrt{\lambda^{2}+\left(1+\frac{1}{\lambda}\right)^{2}},-1-\frac{1}{\lambda}, \lambda, 0\right)\right] \\
& \in \operatorname{cl}\left(\bigcup_{\lambda \in \mathbb{R}} \lambda\left(a_{1}, b_{1}\right)+\left(-K^{*}\right) \times \mathbb{R}^{+}\right) .
\end{aligned}
$$


Since $\sqrt{\lambda^{2}+\left(1+\frac{1}{\lambda}\right)^{2}} x_{1}(t)+\left(1+\frac{1}{\lambda}\right) x_{2}(t)+\lambda x_{3}(t) \geqq 0$ a. e. in $[0,1]$ for any $\left(x_{1}, x_{2}, x_{3}\right) \in K$, the last relation holds. So, the set $\bigcup_{\lambda \in \mathbb{R}} \lambda\left(a_{1}, b_{1}\right)+\left(-K^{*}\right) \times \mathbb{R}^{+}$is not closed.

Using [13, Proposition 2.1], we can prove that the a Slater type constraint qualification implies that the set in the assumption of Theorem 2.2 is closed in $L_{n}^{2}[0,1] \times \mathbb{R}$. In the following proposition, for completeness, we give its proof by following the proof of [13, Proposition 2.1].

Proposition 2.3. Assume that the following constraint qualifications hold:

(i) there exists $\widehat{x}(\cdot) \in$ int $K$ such that

$$
\int_{0}^{1} a_{i}(t)^{T} \widehat{x}(t) d t=b_{i}, i=1, \cdots, m
$$

(ii) $a_{1}, \cdots, a_{m}$ are linearly independent in $L_{n}^{2}[0,1]$.

Then $\Lambda:=\bigcup_{\lambda_{i} \in \mathbb{R}}\left\{\left(\sum_{i=1}^{m} \lambda_{i} a_{i}, \sum_{i=1}^{m} \lambda_{i} b_{i}\right)\right\}+\left(-K^{*}\right) \times \mathbb{R}_{+}$is closed in $L_{n}^{2}[0,1] \times \mathbb{R}$.

Proof. Since $a_{1}, \cdots, a_{m}$ are linearly independent in $L_{n}^{2}[0,1]$, it follows from the lemma on the biorthogonal basis in [14] that there exists $a_{1}^{*}, \cdots, a_{n}^{*} \in L_{n}^{2}[0,1]$ such that

$$
\int_{0}^{1} a_{i}^{*}(t)^{T} a_{j}(t) d t= \begin{cases}0, & i \neq j \\ 1, & i=j .\end{cases}
$$

Let $g_{i}(x)=\int_{0}^{1} a_{i}(t)^{T} x(t) d t, i=1, \cdots, m$, and let $G(x)=\left(\begin{array}{c}g_{1}(x) \\ \cdots \\ g_{m}(x)\end{array}\right)$. Then $G$ is a continuous linear mapping from $L_{n}^{2}[0,1]$ to $\mathbb{R}^{n}$. For any $r=\left(r_{1}, \cdots, r_{m}\right)^{T} \in \mathbb{R}^{m}$,

$$
\begin{aligned}
G\left(\sum_{i=1}^{m} r_{i} a_{i}^{*}\right) & =\left(\begin{array}{c}
\int_{0}^{1} a_{1}(t)^{T} \sum_{i=1}^{m} r_{i} a_{i}^{*}(t) d t \\
\cdots \\
\int_{0}^{1} a_{m}(t)^{T} \sum_{i=1}^{m} r_{i} a_{i}^{*}(t) d t
\end{array}\right) \\
& =\left(\begin{array}{c}
r_{1} \\
\cdots \\
r_{m}
\end{array}\right) \\
& =r .
\end{aligned}
$$

So, $G$ is surjective. By the open mapping theorem, $G(\operatorname{int} K)$ is open. By (i), $\left(\begin{array}{c}b_{1} \\ \cdots \\ b_{m}\end{array}\right) \in G(\operatorname{int} K)$. Let $h_{i}(x)=\int_{0}^{1} a_{i}(t)^{T} x(t) d t-b_{i}, x \in L_{n}^{2}[0,1], i=1, \cdots, m$ and $H(x)=\left(\begin{array}{c}h_{1}(x) \\ \cdots \\ h_{m}(x)\end{array}\right)$. Then $0 \in$ $H(\operatorname{int} K)$, since $H(\operatorname{int} K)$ is open, $0 \in \operatorname{int} H(K)$. Let $\Lambda=\bigcup_{\lambda_{i} \in \mathbb{R}}$ epi $\left(\sum_{i=1}^{m} \lambda_{i} h_{i}\right)^{*}+$ epi $\delta_{K}^{*}$. Observe that epi $\delta_{\triangle}^{*}$ is closed. To see that the set $\Lambda$ is closed, it is sufficient to prove that $\Lambda=$ epi $\delta_{\triangle}^{*}$. Let $(v, \alpha) \in \Lambda$. Then there exist $\lambda_{i} \in \mathbb{R}, i=1, \cdots, m,\left(v_{1}, \alpha_{1}\right) \in \operatorname{epi}\left(\sum_{i=1}^{m} \lambda_{i} h_{i}\right)^{*}$ and $\left(v_{2}, \alpha_{2}\right) \in$ epi $\delta_{K}^{*}$ such that $v=v_{1}+v_{2}$ and $\alpha=\alpha_{1}+\alpha_{2}$. Since $\left.\sum_{i=1}^{m} \lambda_{i} h_{i}\right)^{*}\left(v_{1}\right) \leqq \alpha_{1}$ and $\delta_{K}^{*}\left(v_{2}\right) \leqq \alpha_{2}$, $v_{1}(x)-\sum_{i=1}^{m} \lambda_{i} h_{i}(x) \leqq \alpha_{1}$ for any $x \in L_{n}^{2}[0,1]$ and $v_{2}(x) \leqq \alpha_{2}$ for any $x \in K$. Thus $v_{1}(x) \leqq$ 
$\alpha_{1}$ and $v_{2}(x) \leqq \alpha_{2}$ for any $x \in \triangle$. So, $\left(v_{1}+v_{2}\right)(x) \leqq \alpha_{1}+\alpha_{2}$, for any $x \in \triangle$, i.e., $v(x) \leqq$ $\alpha$ for any $x \in \triangle$. Hence $(v, \alpha) \in \operatorname{epi} \delta_{\triangle}^{*}$. Thus $\Lambda \subset$ epi $\delta_{\triangle}^{*}$. If $(v, r) \in$ epi $\delta_{\triangle}^{*}$, then $v \in \operatorname{dom} \delta_{\triangle}^{*}$. Let $T=\left\{(p, y) \in \mathbb{R} \times \mathbb{R}^{m} \mid \exists x \in K\right.$ such that $\left.v(x) \geqq p, H(x)=y\right\}$. Then $T$ is a nonempty convex set. Since $\delta_{\triangle}^{*}(v)=\sup _{x \in \triangle} v(x)<+\infty$, for any $\varepsilon>0$, there exists $\widetilde{x} \in \triangle$ such that $\delta_{\triangle}^{*}(v)-\varepsilon<v(\widetilde{x})$. So, $\left(\delta_{\triangle}^{*}(v)-\varepsilon, 0\right) \in T$. But $\left(\delta_{\triangle}^{*}(v)+\varepsilon, 0\right) \notin T$. Thus $\left(\delta_{\triangle}^{*}(v), 0\right)$ is a boundary point of $T$. Since $v$ is continuous, we have that $V:=v^{-1}(r, r)$ is an open neighborhood of 0 for any fixed $r>0$. By assumption (i), there exists $\widehat{x} \in \operatorname{int} K$ such that $H(\widehat{x})=0$. Let $B=(v(\widehat{x})-r-\mu, v(\widehat{x})-$ $r) \times H(\widehat{x}+V) \cap \operatorname{int} K$ for fixed $\mu>0$. Since $H$ is an open mapping, $B$ is open, and for any $x \in(\widehat{x}+V) \cap \operatorname{int} K$,

$$
v(\widehat{x})-r<v(x)<v(\widehat{x})+r .
$$

If $(\alpha, w) \in B$, then there exists $u \in(\widehat{x}+V) \cap$ int $K$ such that $w=H(u)$ and $v(u)>v(\widehat{x})-r>\alpha$. So, $(\alpha, w) \in T$. Thus $B \subset T$ and int $T \neq \emptyset$. Since $\left(\delta_{\triangle}^{*}(v), 0\right)$ is a boundary point of $T$, we have

$$
\left(\delta_{\triangle}^{*}(v), 0\right) \notin \operatorname{int} T .
$$

By the separation theorem, there exists $(\theta, \lambda) \in \mathbb{R} \times \mathbb{R}^{m}$ such that $(\theta, \lambda) \neq(0,0)$, and

$$
\theta \delta_{\triangle}^{*}(v) \leqq \theta p+\lambda^{T} y \text { for any }(p, y) \in T .
$$

If $\theta=0$, then $0 \leqq \lambda^{T} y$ for any $y \in\left\{y \in \mathbb{R}^{m} \mid y=H(x), \quad x \in K\right\}$. Since $0 \in \operatorname{int} H(K), \lambda=0$. This is a contradiction. Hence $\theta \neq 0$. Since $\left(\delta_{\triangle}^{*}(v)-\varepsilon, 0\right) \in T$ for any $\varepsilon>0$, and $\theta \leqq 0$. Thus we may assume that $\theta=-1$. Hence $\delta_{\triangle}^{*}(v) \geqq p-\lambda^{T} y$ for any $(p, y) \in T$. So, $\delta_{\triangle}^{*}(v) \geqq$ $v(x)-\lambda^{T} H(x)$ for any $x \in K$. Thus $\delta_{\triangle}^{*}(v) \geqq v(x)-\sum_{i=1}^{m} \lambda_{i} h_{i}(x)-\delta_{K}(x), \forall x \in L_{n}^{2}[0,1]$. It follows that $\delta_{\triangle}^{*}(v) \geqq\left(\sum_{i=1}^{m} \lambda_{i} h_{i}+\delta_{K}\right)^{*}(v)$. Since $(v, r) \in$ epi $\delta_{\triangle}^{*}$, then $r \geqq \delta_{\triangle}^{*}(v) \geqq\left(\sum_{i=1}^{m} \lambda_{i} h_{i}+\delta_{K}\right)^{*}(v)$. Thus $(v, r) \in \operatorname{epi}\left(\sum_{i=1}^{m} \lambda_{i} h_{i}+\delta_{K}\right)^{*}$. Since $\lambda_{i} h_{i}$ is continuous, then $(v, r) \in \operatorname{epi}\left(\sum_{i=1}^{m} \lambda_{i} h_{i}\right)^{*}+\mathrm{epi} \delta_{K}^{*}$. Hence epi $\sum_{i=1}^{m} \delta_{\triangle}^{*} \subset \Lambda$. Consequently, $\Lambda=$ epi $\sum_{i=1}^{m} \delta_{\triangle}^{*}$, and hence $\Lambda$ is closed.

Now we give an example to show that the converse of Proposition 2.3 may not be true.

Example 2.2. Consider the following optimization problem:

$$
\begin{array}{ll}
\text { Minimize } & \int_{0}^{1} x(t) d t \\
\text { subject to } & x \in K, \\
& \int_{0}^{1} t x(t) d t=\frac{1}{2},
\end{array}
$$

where $K=\{\alpha a \mid \alpha \geqq 0\}$ and $a(t)=t$ for $t \in[0,1]$. Then $\triangle:=\left\{x \in K \mid \int_{0}^{1} t x(t) d t=\frac{1}{2}\right\}=\{q\}$, where $q(t)=\frac{3}{2} t$ for $t \in[0,1]$. Let $\varepsilon>0$ be any given, and let $\beta$ be a positive number with $\beta^{2}<5 \varepsilon^{2}$. Then

$$
\left[\int_{0}^{1}\left(\beta t^{2}+t-t\right)^{2} d t\right]^{\frac{1}{2}}=\sqrt{\frac{\beta^{2}}{5}}<\varepsilon .
$$

We can check that there does not exist $\alpha \geq 0$ such that

$$
\left[\int_{0}^{1}\left(\beta t^{2}+t-\alpha t\right)^{2} d t\right]^{\frac{1}{2}}=0
$$


Hence $p \notin K$, where $p(t)=\beta t^{2}+t$ for $t \in[0,1]$. Thus int $K=\emptyset$. Let $\Lambda=\bigcup_{\lambda \in \mathbb{R}}\left\{\lambda\left(a, \frac{1}{2}\right)\right\}+$ $\left(-K^{*}\right) \times \mathbb{R}_{+}$. Now we will show that $\Lambda$ is closed. Let $\left(p_{n}, r_{n}\right) \in \Lambda$ converge to $\left(p^{*}, r^{*}\right) \in$ $L^{2}[0,1] \times \mathbb{R}$. Then there exist $\lambda_{n} \in \mathbb{R}, k_{n}^{*} \in K^{*}$ and $\rho_{n} \in \mathbb{R}_{+}$such that

$$
p_{n}=\lambda_{n} a-k_{n}^{*} \text { and } r_{n}=\frac{1}{2} \lambda_{n}+\rho_{n} .
$$

The first case that $\left\{\lambda_{n}\right\}$ is bounded:

We may assume that $\lambda_{n}$ converges to $\widehat{\lambda}$. Moreover $k_{n}^{*}\left(=\lambda_{n} a-p_{n}\right)$ converges to $\widehat{\lambda} a-p^{*}$ and $\rho_{n}\left(=-\frac{1}{2} \lambda_{n}+r_{n}\right)$ converges to $-\frac{1}{2} \widehat{\lambda}+r^{*}$. Since $K^{*}$ is closed, then $\widehat{\lambda} a-p^{*} \in K^{*}$. Also, $-\frac{1}{2} \widehat{\lambda}+r^{*} \in \mathbb{R}_{+}$. Hence $\left(p^{*}, r^{*}\right) \in \Lambda$.

The second case that $\left\{\lambda_{n}\right\}$ is unbounded:

We may assume that $\lambda_{n} \rightarrow+\infty$ or $\lambda_{n} \rightarrow-\infty$. The case that $\lambda_{n} \rightarrow+\infty$ does not occur since $r_{n}$ converges to $r^{*}$. Consider the case that $\lambda_{n} \rightarrow-\infty$,

$$
\begin{aligned}
K^{*} & =\left\{z \in L^{2}[0,1] \mid \int_{0}^{1} z(t) \alpha a(t) d t \geqq 0 \quad \forall \alpha \geqq 0\right\} \\
& =\left\{z \in L^{2}[0,1] \mid \int_{0}^{1} z(t) a(t) d t \geqq 0\right\} .
\end{aligned}
$$

Since $\lambda_{n} a-p_{n}=k_{n}^{*} \in K^{*}, \int_{0}^{1}\left(\lambda_{n} a(t)-p_{n}(t)\right) t d t \geqq 0$ and $\int_{0}^{1} \lambda_{n} t^{2} d t \geqq \int_{0}^{1} p_{n}(t) t d t$. Letting $n \rightarrow$ $+\infty$, we have that $\int_{0}^{1} p_{n}(t) t d t \rightarrow \int_{0}^{1} p^{*}(t) t d t$ and $\left\{\int_{0}^{1} p_{n}(t) t d t\right\}$ is bounded, but $\int_{0}^{1} \lambda_{n} t^{2} d t \rightarrow-\infty$. This is a contradiction. So, the case that $\lambda_{n} \rightarrow-\infty$ does not occur. Consequently, $\Lambda$ is closed.

\section{Duality Theorems}

Using Theorem 2.1, we formulate a dual problem for (P) as follows:

$$
\begin{array}{cl}
\text { Maximize } & \gamma \\
\text { subject to } & c-\gamma d+\lim _{n \rightarrow \infty}\left(\sum_{i=1}^{m} y_{i}^{n} a_{i}-k_{n}^{*}\right)=0, \\
& \limsup _{n \rightarrow \infty} \sum_{i=1}^{m} y_{i}^{n} b_{i} \leqq \alpha-\beta \gamma, \\
& \left\{y_{i}^{n}\right\} \subset \mathbb{R}, i=1, \cdots, m,\left\{k_{n}^{*}\right\} \subset K .
\end{array}
$$

Theorem 3.1. (Weak duality) Suppose that, for any $x \in \triangle, \int_{0}^{1} d(t)^{T} x(t) d t+\beta>0$. Let $x$ be feasible for $(P)$, and let $\left(\gamma,\left\{y_{i}^{n}\right\},\left\{k_{n}^{*}\right\}\right)$ be feasible for $(D)$. Then

$$
\frac{\int_{0}^{1} c(t)^{T} x(t) d t+\alpha}{\int_{0}^{1} d(t)^{T} x(t) d t+\beta} \geqq \gamma .
$$

Proof. Let $x$ be feasible for (P), and let $\left(\gamma,\left\{y_{i}^{n}\right\},\left\{k_{n}^{*}\right\}\right)$ be feasible for (D). Suppose to the contrary that

$$
\frac{\int_{0}^{1} c(t)^{T} x(t) d t+\alpha}{\int_{0}^{1} d(t)^{T} x(t) d t+\beta}<\gamma
$$


Since $\int_{0}^{1} d(t)^{T} x(t) d t+\beta>0$, we have

$$
\int_{0}^{1} c(t)^{T} x(t) d t+\alpha-\gamma \int_{0}^{1} d(t)^{T} x(t) d t-\gamma \beta<0
$$

and

$$
\int_{0}^{1}[c(t)-\gamma d(t)]^{T} x(t) d t<\gamma \beta-\alpha
$$

Hence

$$
\lim _{n \rightarrow \infty}\left[-\sum_{i=1}^{m} \int_{0}^{1} y_{i}^{n} a_{i}(t)^{T} x(t) d t+\int_{0}^{1} k_{n}^{*}(t)^{T} x(t) d t\right]<\gamma \beta-\alpha
$$

So, we have

$$
\begin{aligned}
\gamma \beta-\alpha & >\lim _{n \rightarrow \infty}\left[-\sum_{i=1}^{m} y_{i}^{n} b_{i}+\int_{0}^{1} k_{n}^{*}(t)^{T} x(t) d t\right] \\
& \geqq \liminf _{n \rightarrow \infty}\left[-\sum_{i=1}^{m} y_{i}^{n} b_{i}\right]+\liminf _{n \rightarrow \infty} \int_{0}^{1} k_{n}^{*}(t)^{T} x(t) d t .
\end{aligned}
$$

Since $\int_{0}^{1} k_{n}^{*}(t)^{T} x(t) d t \geqq 0, \gamma \beta-\alpha>\liminf _{n \rightarrow \infty}\left[-\sum_{i=1}^{m} y_{i}^{n} b_{i}\right] \alpha-\gamma \beta<\limsup _{n \rightarrow \infty} \sum_{i=1}^{m} y_{i}^{n} b_{i}$. This is a contradiction. Consequently,

$$
\frac{\int_{0}^{1} c(t)^{T} x(t) d t+\alpha}{\int_{0}^{1} d(t)^{T} x(t) d t+\beta} \geqq \gamma
$$

Theorem 3.2. (Strong duality) Suppose that, for any $x \in \triangle, \int_{0}^{1} d(t)^{T} x(t) d t+\beta>0$. Let $\bar{x}$ be an optimal solution of $(P)$ and let $\bar{\gamma}=\frac{\int_{0}^{1} c(t)^{T} \bar{x}(t) d t+\alpha}{\int_{0}^{1} d(t)^{T} \bar{x}(t) d t+\beta}$. Then there exist $\bar{y}_{i}^{n} \in \mathbb{R}, i=1, \cdots, m$ and $\bar{k}_{n}^{*} \in K^{*}$ such that $\left(\bar{\gamma},\left\{\bar{y}_{i}^{n}\right\},\left\{\bar{k}_{n}^{*}\right\}\right)$ is an optimal solution of $(D)$.

Proof. Let $\bar{x} \in \triangle$ be an optimal solution of (P). By Theorem 2.1, there exist $\bar{y}_{i}^{n} \in \mathbb{R}, i=1, \cdots, m$ and $\bar{k}_{n}^{*} \in K^{*}$ such that

$$
\begin{aligned}
& c-q(\bar{x}) d+\lim _{n \rightarrow \infty}\left(\sum_{i=1}^{m} \bar{y}_{i}^{n} a_{i}-\bar{k}_{n}^{*}\right)=0 \\
& \text { and } \lim _{n \rightarrow \infty} \int_{0}^{1} \bar{k}_{n}^{*}(t)^{T} \bar{x}(t) d t=0,
\end{aligned}
$$

where $q(\bar{x})=\frac{\int_{0}^{1} c(t)^{T} \bar{x}(t) d t+\alpha}{\int_{0}^{1} d(t)^{T} \bar{x}(t) d t+\beta}$. Then $\bar{\gamma}=q(\bar{x})$ and

$$
\int_{0}^{1}[c(t)-\bar{\gamma} d(t)]^{T} \bar{x}(t) d t+\lim _{n \rightarrow \infty}\left[\sum_{i=1}^{m} \int_{0}^{1} \bar{y}_{i}^{n} a_{i}(t)^{T} \bar{x}(t) d t-\int_{0}^{1} \bar{k}_{n}^{*}(t)^{T} \bar{x}(t) d t\right]=0 .
$$

Since $\bar{\gamma}=q(\bar{x}), \int_{0}^{1}[c(t)-\bar{\gamma} d(t)]^{T} \bar{x}(t) d t=\bar{\gamma} \beta-\alpha$,

$$
\bar{\gamma} \beta-\alpha+\lim _{n \rightarrow \infty}\left(\sum_{i=1}^{m} \int_{0}^{1} \bar{y}_{i}^{n} a_{i}(t)^{T} \bar{x}(t) d t-\int_{0}^{1} \bar{k}_{n}^{*}(t)^{T} \bar{x}(t) d t\right)=0 .
$$


Since $\int_{0}^{1} a_{i}(t)^{T} \bar{x}(t) d t=b_{i}, i=1, \cdots, m$ and $\lim _{n \rightarrow \infty} \int_{0}^{1} \bar{k}_{n}^{*}(t)^{T} \bar{x}(t) d t=0$,

$$
\lim _{n \rightarrow \infty} \sum_{i=1}^{m} \bar{y}_{i}^{n} b_{i}=\alpha-\bar{\gamma} \beta .
$$

By (3.1) and (3.2), we have that $\left(\bar{\gamma},\left\{\bar{y}_{i}^{n}\right\},\left\{\bar{k}_{n}^{*}\right\}\right)$ is feasible for (D). In view of the weak duality theorem, we conclude that $\left(\bar{\gamma},\left\{\bar{y}_{i}^{n}\right\},\left\{\bar{k}_{n}^{*}\right\}\right)$ is an optimal solution of (D).

Using Theorem 2.2, we can formulate a dual problem (CQD) ([8]) for (P) as follows:

$$
\begin{array}{ll}
\text { Maximize } & \gamma \\
\text { subject to } & c+\sum_{i=1}^{m} y_{i}^{n} a_{i}-\gamma d \in K^{*} \\
& \beta \gamma+b^{T} y \leqq \alpha, \gamma \in \mathbb{R}, y \in \mathbb{R}^{m} .
\end{array}
$$

Theorem 3.3. [8] (Weak duality) Assume that, for any $x \in \triangle, \int_{0}^{1} d(t)^{T} x(t) d t+\beta>0$. Let $x$ be feasible for $(P)$, and let $(\gamma, y)$ be feasible for $(D)$. Then

$$
\frac{\int_{0}^{1} c(t)^{T} x(t) d t+\alpha}{\int_{0}^{1} d(t)^{T} x(t) d t+\beta} \geqq \gamma .
$$

We can prove the following strong theorem.

Theorem 3.4. (Strong duality) Let $\bar{x}$ be an optimal solution of $(P)$ and assume that, for any $x \in \triangle, \int_{0}^{1} d(t)^{T} x(t) d t+\beta>0$. Suppose that the set $\bigcup_{\lambda_{i} \in \mathbb{R}} \sum_{i=1}^{m}\left\{\lambda_{i}\left(a_{i}, b_{i}\right)\right\}+\left(-K^{*}\right) \times \mathbb{R}_{+}$is closed in $L_{n}^{2}[0,1] \times \mathbb{R}$. Let $\bar{\gamma}=\frac{\int_{0}^{1} c(t)^{T} \bar{x}(t) d t+\alpha}{\int_{0}^{1} d(t)^{T} \bar{x}(t) d t+\beta}$. Then there exist $\bar{y} \in \mathbb{R}^{m}$ such that $(\bar{\gamma}, \bar{y})$ is an optimal solution of $(D)$.

Theorem 3.5. [8] (Converse duality) Assume that, for any $x \in \triangle, \int_{0}^{1} d(t)^{T} x(t) d t+\beta>0$, and that $\triangle$ is bounded. Further assume that there exist $\widetilde{y} \in \mathbb{R}^{m}$ and $\widetilde{\gamma} \in \mathbb{R}$ such that $c+\sum_{i=1}^{m} \widetilde{y}_{i} a_{i}-$ $\widetilde{\gamma} d \in \operatorname{int}^{*}$ and $\beta \widetilde{y}+b^{T} \widetilde{y}<\alpha$. If $(\bar{\gamma}, \bar{y})$ is an optimal solution of $(D)$, then there exists $\bar{x} \in \triangle$ such that $\bar{x}$ is an optimal solution of $(P)$, and $\bar{\gamma}=\frac{\int_{0}^{1} c(t)^{T} \bar{x}(t) d t+\alpha}{\int_{0}^{1} d(t)^{T} \bar{x}(t) d t+\beta}$.

\section{Acknowledgments}

This work was supported by the National Research Foundation of Korea (NRF) grant funded by the Korea government (MSIT) (NRF-2017R1E1A1A03069931).

\section{REFERENCES}

[1] V. Jeyakumar, G.M. Lee, N. Dinh, New sequential Lagrange multiplier conditions characterizing optimality without constraint qualification for convex programs, SIAM J. Optim. 14 (2003), 534-547.

[2] V. Jeyakumar, Z.Y. Wu, G.M. Lee, N. Dinh, Liberating the subgradient optimality conditions from constraint qualifications, J. Global Optim. 36 (2006), 127-137.

[3] V. Jeyakumar, G. Li, Strong duality in robust convex programming: complete characterizations, SIAM J. Optim. 20 (2010), 3384-3407.

[4] G.M. Lee, K.B. Lee, On optimality conditions for abstract convex vector optimization problems, J. Korean Math. Soc. 44 (2007), 971-985. 
[5] J.H. Lee, G.M. Lee, On Sequential optimality theorems for convex optimization problems, Linear Nonlinear Anal. 2 (2017), 155-170.

[6] B.D. Craven, Fractional Programming, Heldermann Verlag, Berlin, 1988.

[7] B.D. Craven, B. Mond, The dual of a fractional linear program, J. Math. Anal. Appl. 42 (1973), 507-512.

[8] G.S. Kim, M.H. Kim, G.M. Lee, On duality theorems for linear fractional optimization problems involving integral functions, Linear Nonlinear Anal. 6 (2020), 439-445.

[9] M.H. Kim, G.S. Kim, G.M. Lee, On semidefinite linear fractional optimization problems, J. Nonlinear Convex Anal. 22 (2021), 1297-1310.

[10] G.S. Kim, M.H. Kim, G.M. Lee, On optimality and dulaity for second-order cone linear fractional optimization problems, in press.

[11] A. Dhara, J. Duatta, Optimality Conditions in Convex Optimization: A Finite-Dimensional View, Taylor and Francis Group, LLC, 2012.

[12] V. Jeyakumar, G.M. Lee, N. Dinh, Characterization of solution sets of convex vector minimization problems, Eur. J. Oper. Res 174 (2006), 1380-1395.

[13] V. Jeyakumar, N. Dinh, G.M. Lee, A new closed cone constraint qualification for convex optimization, Applied Mathematics Research Report AMR0416, University of New South Wales, 2003.

[14] A.D. Ioffe, V.M. Tihomirov, Theory of Extremal Problems, North-Holland Publishing Company, 1979. 